\title{
Genèse \\ d'ondes internes dans un milieu à deux couches
}

\author{
par M. Crépon \\ Laboratoire d'Océanographie Physique \\ du Muséum, Paris
}

\section{Introduction}

Depuis 1964, l'équipe du Laboratoire d'Océanographie Physique du Muséum National d'Histoire Naturelle a observé, à partir de la bouée-laboratoire du CNEXO, des oscillations de la thermocline d'une amplitude de quelques mètres et de période légèrement inférieure à la période de Coriolis locale (J. Gonella, M. Crépon et F. Madelain, 1969). En juillet 1973, un upwelling côtier très rapide et très important (chute de $10^{\circ} \mathrm{C}$ en 24 heures de la température de la mer) a été observé par la même équipe à bord de la bouée-laboratoire Bohra II ancrée au sud du Lavandou (J. Gonella, 1974). On se propose de donner ici un schéma mathématique simple rendant compte de ces phénomènes.

\section{Formulation du problème}

Soit un océan formé de deux liquides superposés de densités différentes. Etant donné les ordres de grandeur des paramètres observés (oscillations verticales de quelques mètres, périodes voisines de la période d'inertie), une analyse dimensionnelle montre que les équations peuvent être linéarisées dans l'hypothèse des ondes longues (pression hydrostatique).

On admet que le couplage entre le fluide supérieur (paramètres indicés par le chiffre 1) et le fluide inférieur (paramètres indicés par le chiffre 2, voir fig. 1) est dû uniquement aux forces de pression. Ce modèle schématise bien l'océan réel lorsqu'il y a une thermocline accentuée, donc une forte discontinuité de densité à ce niveau; on en trouve une justification dans l'analyse des mesures de courant faites à bord de la bouée-laboratoire par l'équipe du Laboratoire d'Océanographie du Muséum et qui sont présentées par J. Gonella, M. Crépon et F. Madelain (1969) et M. Crépon et D. Chouchan (1974).

Les équations linéarisées de mouvement et de continuité s'écrivent alors dans le fluide supérieur :

$$
\left.\begin{array}{c}
\left(\frac{\partial}{\partial t}+\nu_{1}\right) \vec{u}_{1}+\overrightarrow{f z} \times \vec{u}_{1}=-h_{1} \nabla \varphi_{1}+\frac{\vec{\tau}}{\rho_{1}} \\
\frac{\partial}{\partial t}\left(\zeta_{1}-\zeta_{2}\right)+\nabla \vec{u}_{1}=0
\end{array}\right\}
$$

avec : $\varphi_{1}=g\left(\zeta_{1}-\bar{\zeta}\right)$

et, dans le fluide inférieur:

$$
\left.\begin{array}{c}
\left(\frac{\partial}{\partial t}+\nu_{2}\right) \vec{u}_{2}+f \vec{z} \times \vec{u}_{2}=-h_{2} \nabla \varphi_{2} \\
\frac{\partial}{\partial t} \zeta_{2}+\nabla \vec{u}_{2}=0
\end{array}\right\}
$$

avec :

$$
\varphi_{2}=g \frac{\rho_{1}}{\rho_{2}}\left(\zeta_{1}-\bar{\zeta}\right)+g \frac{\Delta \rho}{\rho_{2}} \zeta_{2}
$$

où :

$\vec{u}_{i}(x, y, t)$ désigne la vitesse intégrée (ou transport de masse) selon la verticale dans la couche $i$;

$\zeta_{i}(x, y, t)$ la surélévation à la surface libre (indice 1) ou à l'interface (indice 2); 
$\bar{\zeta}(x, y, t)$ l'inverse de la pression atmosphérique exprimée dans la même unité que la surélévation;

$f \quad$ le paramètre de Coriolis supposé constant;

$\ddot{z} \quad$ le vecteur unité selon la verticale ascendante;

$h_{i}(x, y) \quad$ l'épaisseur du milieu $i$;

$\rho_{i} \quad$ la densité du milieu $i$;

$\vec{\tau}(x, y, t)$ l'action du vent (wind stress);

$\nabla \quad$ l'opérateur horizontal $(\partial / \partial x, \partial / \partial y)$;

$\nu_{i} \quad$ un coefficient de dissipation d'énergie dans le milieu $i$.

La résolution du système formé par les équations (1) et (2) est difficile. On peut la simplifier en se servant de transformations intégrales.

On suppose que les mouvements partent du repos, que $\vec{u}_{i}, \zeta_{i}, \bar{\zeta}$ et $\vec{\tau}$ sont nuls pour $t<0$. On résout alors le système (1) et (2) au moyen de la transformée de Laplace sur le temps. Soit $\vec{U}_{i}, Z_{i}, \bar{Z}, \Phi_{i}, \vec{T}$ les transformées de Laplace, respectivement de $\vec{u}_{i}, \zeta_{i}, \bar{\zeta}, \varphi_{i}, \vec{\tau}$, telles que:

$$
\vec{U}_{i}(x, y, p)=\int_{0}^{\infty} e^{-p t} \vec{u}_{i}(x, y, t) d t
$$

On obtient par combinaisons linéaires des transformées des équations (1) et (2) le système (3) qui associé aux conditions aux limites permet d'avoir $\Phi_{1}$ et $\Phi_{2}$

$$
\begin{gathered}
\Phi_{1}-\Phi_{2}-g \frac{\Delta \rho}{\rho}\left[\frac{h_{1}}{p^{2} K_{1}^{2}} \nabla^{2} \Phi_{1}-\frac{1}{\rho_{1} p^{2} K_{1}^{2}}\right. \\
\left.\left(\nabla \vec{T}+\frac{f}{p+\nu_{1}} \nabla \times \vec{T}\right)-\bar{Z}\right]=0 \\
\Phi_{2}-\frac{\rho_{1}}{\rho_{2}} \Phi_{1}-g \frac{\Delta \rho}{\rho_{2}} \frac{h_{2}}{p^{2} K_{2^{2}}} \nabla^{2} \Phi_{2}=0
\end{gathered}
$$

avec :

$$
K_{i}{ }^{2}=\left[\left(p+\nu_{i}\right)^{2}+f^{2}\right] /\left[p\left(p+\nu_{i}\right)\right]
$$

Pour simplifier les calculs, on pose $\nu_{1}=\nu_{2}=\nu$ d'où $K_{1}=K_{2}=K$.

L'inversion de la transformation de Laplace permet de connaître $\varphi_{1}$ et $\varphi_{2}$ et à partir des systèmes (1) et (2) d'obtenir $\zeta_{1}$ et $\zeta_{2}$ et $\vec{u}_{1}$ et $\vec{u}_{2}$.

\section{Vent constant dans l'espace soufflant sur un océan semi-infini}

Soit un océan s'étendant sur le demi-plan $x \geqslant 0$ et borné par un mur vertical d'équation $x=0$.

On suppose que la tension du vent sur le demi-plan est de la forme:

$$
\vec{\tau}=\left(\tau_{x} \vec{x}+\tau_{y} \vec{y}\right) Y(x) Y(t)
$$

où $Y(x)$ est la fonction unité d'Heaviside;

$\tau_{x}$ et $\tau_{y}$, composantes de la tension du vent respecti- vement sur $o x$ et $o y$, sont des constantes indépendantes de $x, y$ et $t$.

On a :

$$
\left.\begin{array}{rl}
\operatorname{div} \tau & =\nabla \tau=\tau_{x} \delta(x) \\
\operatorname{rot} \tau & =\nabla \times \tau=\tau_{y /} \delta(x)
\end{array}\right\}
$$

De la première équation du système (3), on remarque que l'on peut partager les solutions du problème en deux classes : la classe $\mathrm{A}$, qui concerne les vents dont la tension est purement divergente et dont le rotationnel est nul [cas d'un vent perpendiculaire au rivage, par exemple d'après (5)] et la classe $B$, qui concerne les vents dont la tension est purement rotationnelle et dont la divergence est nulle [cas d'un vent parallèle au rivage, par exemple d'après (5)]. Si la durée de la perturbation météorologique est supérieure à la période de Coriolis, une analyse dimensionnelle faite sur l'équation (3) montre que la réponse dépend principalement du rotationnel du vent. On doit donc s'attendre, en tenant compte de (5) que les effets d'une côte soient beaucoup plus importants pour un vent soufflant parallèlement au rivage que pour un vent soufflant perpendiculairement au rivage.

\section{A - Ełude des surélévations}

La résolution du système (3), compte tenu de (5) et des conditions aux limites (composante de la vitesse intégrée selon $o x$ nulle en $x=0$ ), donne pour les surélévations :

$$
\begin{gathered}
\zeta_{1}=\frac{1}{\rho_{1} c_{1} f}\left[\zeta\left(x / c_{1}, t\right)+\frac{h_{2}}{h_{1}} \frac{c_{2}}{c_{1}} \zeta\left(x / c_{2}, t\right)\right] . \\
{\left[\tau_{x}+\underset{(f t)}{*} \tau_{y,} e^{-v t} Y(f t)\right]}
\end{gathered}
$$$$
\begin{gathered}
\zeta_{2}=\frac{1}{\rho_{1} c_{1} f}\left[\frac{h_{2}}{h_{1}+h_{2}} \zeta\left(x / c_{1}, t\right)-\sqrt{\frac{h_{2}}{h_{1} \Delta \rho / \rho}} \zeta\left(x / c_{2}, t\right)\right] . \\
{\left[\tau_{x}+\underset{(f t)}{*} \tau_{y} e^{-\eta t} Y(f t)\right]}
\end{gathered}
$$$$
\text { où } \quad c_{1}=\sqrt{g\left(h_{1}+h_{2}\right)} \quad, \quad c_{2} \simeq \sqrt{g \frac{h_{1} h_{2}}{h_{1}+h_{2}} \frac{\Delta \rho}{\rho}}
$$

$$
\zeta\left(x / c_{i}, t\left[\frac{1}{p^{2} K} e^{-p K i x l c_{i}}\right.\right.
$$

et $\div$ désigne le produit de convolution.

Chacune des surélévations $\zeta_{1}$ et $\zeta_{2}$ est la somme de deux surélévations dont le front se propage à partir du plan $x=0$, respectivement avec la célérité $c_{1}$ et la célérité $c_{2}$.

Dans le cas où $\nu=0$, on a :

$$
\begin{gathered}
\zeta\left(x / c_{i}, t\right)=\int_{0}^{f t} Y\left(t-x / c_{i}\right) J_{0} f \sqrt{t^{2}-x^{2} / c_{i}^{2}} d(f t) \\
\zeta\left(x / c_{i}, t\right) \underset{(f t)}{\underset{(f t)}{*}} Y(f t)=\int_{0}^{f t} \zeta\left(x / c_{i}, t\right) d(f t)
\end{gathered}
$$


où $J_{n}(x)$ désigne la fonction de Bessel de première espèce d'ordre $n$.

Dans l'expression de $\zeta_{1}$, les termes liés à la vitesse de propagation $c_{1}$ représentent la surélévation d'un océan à une couche lorsque souffle un vent de type (4).

Les surélévations les plus importantes sont données par: les termes de $\zeta_{2}$ liés à la vitesse de propagation $c_{2}$. On a création d'ondes internes de grande amplitude, dont le front se propage à la célérité $c_{2}$.

Les fonctions qui caractérisent les surélévations et qui sont du type (7) ou (8) sont les mêmes que celles obtenues par A. Cahn (1945) pour la résolution du problème de l'ajustement géostrophique avec dépendance du temps.

On a tracé, sur la figure 2, la fonction (7) qui représente, dans le cas où $\nu=0$, la surélévation de la surface libre ou de l'interface lorsque le vent est perpendiculaire à la côte, ceci pour différentes valeurs du rapport $x f / c_{i}$ (distance à la côte sur rayon de déformation).

L'amplitude des oscillations passe de 0,5 pour $x f / c_{i}=0$ à 0,15 pour $x f / c_{i}=10$. La période des oscillations, inférieure à $2 \pi / f$ au démarrage, tend vers $2 \pi / f$ lorsque $t$ croît, tandis que leur amplitude diminue.

La fonction (8), qui représente la surélévation de la surface libre ou de l'interface lorsque le vent est parallèie à la côte, a des caractéristiques analogues lorsque $x f / c_{i} \gg 1$. Son comportement lorsque $t$ tend vers l'infini est toutefois très différent.

On a là un processus de formation et de propagation des ondes internes à partir de la côte, qui décrit de façon relativement satisfaisante les ondes internes de période voisine de la période d'inertie que l'on observe à la bouéelaboratoire du CNEXO. En effet, ces ondes ont toujours des périodes légèrement inférieures à la période d'inertie $2 \pi / f$. Leur amplitude, qui est de l'ordre de quelques mètres, coincide avec celle que donne le calcul pour les conditions régnant à la bouée :

$$
\begin{gathered}
\left(h_{1}=25 \mathrm{~m}, h_{2}=2500 \mathrm{~m}, c_{1}=165 \mathrm{~m} / \mathrm{sec} .,\right. \\
c_{2}=0,5 \mathrm{~m} / \mathrm{sec} ., x f / c_{2}=10, \Delta \rho / \rho=10^{-3}
\end{gathered}
$$

lorsque l'on prend pour tension du vent la forme:

$$
\vec{\tau}=2.10^{-3}|w| \vec{w}
$$

où $\vec{w}$ est la vitesse du vent exprimée en $\mathrm{m} / \mathrm{sec}$. La formation à la côte et la propagation très lente du front expliquent le retard considérable que l'on observe parfois entre le début du vent et leur apparition à la bouée-laboratoire, retard qui dépasse la journée durant l'opération COFRASOV I (Crépon et al., 1972).

A partir des propriétés des transformées de Laplace (W. Sutton, 1934), on calcule facilement le développement asymptotique de $\zeta\left(x / c_{i}, t\right)$ pour $t$ grand. On obtient, si $\nu=0$ :

$$
\zeta\left(x / c_{i}, t\right)=e^{-x f / c_{i}}+O\left(\frac{\sin (f t-\pi / 4)}{(f t)^{1 / 2}}\right)
$$

Les vents perpendiculaires au rivage créent des surélévations qui restent bornées lorsque $t$ croît. La période des oscillations tend vers $2 \pi / f$ lorsque $t$ croît, tandis que leur amplitude décrôit comme $(f t)^{-1 / 2}$.
De même, on obtient si $\nu=0$ :

$$
\zeta\left(x / c_{i}, t\right) \underset{(f t)}{\underset{\sim}{\longrightarrow}} Y(f t)=e^{-x f / c_{\imath}}(f t)+O\left(\frac{\cos (f t-\pi / 4)}{(f t)^{1 / 2}}\right)
$$

Les vents parallèles au rivage créent des surélévations à la surface libre et à l'interface, qui croissent comma $e^{-x f / c_{i}}(f t)$. La vitesse de croissance est maximum au rivage. D'après (6), l'amplitude des surélévations à l'interface étant de beaucoup supérieure à l'amplitude des surélévations de la surface libre, le liquide inférieur peut très rapidement atteindre la surface libre. On a là un processus d'upwelling qui est schématisé sur la figure 3 . On peut expliquer ainsi l'upwelling mis en évidence en juillet 1973 par J. Gonella (1974) sur la côte provençale par fort vent de Mistral. J. Gonella observe une chute de température de $10^{\circ}$ en 24 heures, chiffres qui cadrent parfaitement avec l'application de (6) et de (9).

Les équations (6) et (9) cessent d'être valides lorsque le temps $t$ augmente et que les surélévations deviennent grandes par rapport à $h_{1}$. Il faut alors faire intervenir la nonlinéarité des équations et les termes de dissipation. Le schéma ci-dessus donne une interprétation physique et qualitative de l'upwelling. Il ne peut en aucun cas modéliser le phénomène.

Si l'on suppose que les dissipations sont linéaires $(\nu \neq 0)$, on a lorsque $t$ tend vers l'infini :

$$
\begin{aligned}
& \zeta\left(x / c_{i}, t\right) \underset{(f t)}{*} e^{-\nu t} Y(f t) \\
& \quad=\frac{f}{\nu}\left[2\left(\frac{\nu t}{\pi(1+\nu / f)}\right)^{1 / 2}-\frac{x f}{c_{i}}+O\left([\nu t]^{-1 / 2}\right)\right]
\end{aligned}
$$

\section{B - Ełude des viłesses infégrées}

La vitesse intégrée dans la couche supérieure $\vec{u}_{1}(x, t)$ est de la forme:

$$
\begin{aligned}
\vec{u}_{1}(x, t) & =\vec{u}_{L}(t)+\frac{h_{1}}{h_{1}+h_{2}} \vec{u}\left(x / c_{1}, t\right)+ \\
& +\frac{h_{2}}{h_{1}+h_{2}} \vec{u}\left(x / c_{2}, t\right)
\end{aligned}
$$

$u_{L}(\mathrm{t}) \quad$ représente la vitesse intégrée due au vent local (vitesse intégrée qui serait celle obtenue dans le cas d'un océan infini en considérant que le vent, égal au vent local, est constant dans l'espace).

$\vec{u}\left(x / c_{i}, t\right)$ est une vitesse intégrée due à la présence de la côte. Elle est émise à la côte et son front se propage à partir de là à la vitesse $c_{i}$.

On étudie explicitement les vitesses intégrées dans le cas où le vent est parallèle à la côte. En effet, c'est dans ce cas que les surélévations sont maxima, donc que les vitesses sont maxima. Lorsque les dissipations sont négligeables, on $\mathrm{a}$ :

$$
\vec{u}_{L}(t)=\frac{\tau_{y}}{\rho f}[(1-\cos [f t]) \vec{x}+\sin (f t) \vec{y}]
$$




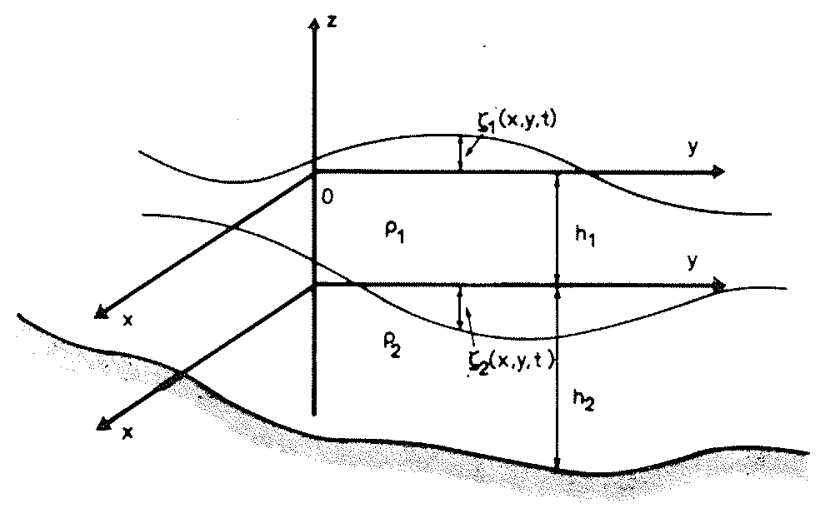

$1 /$
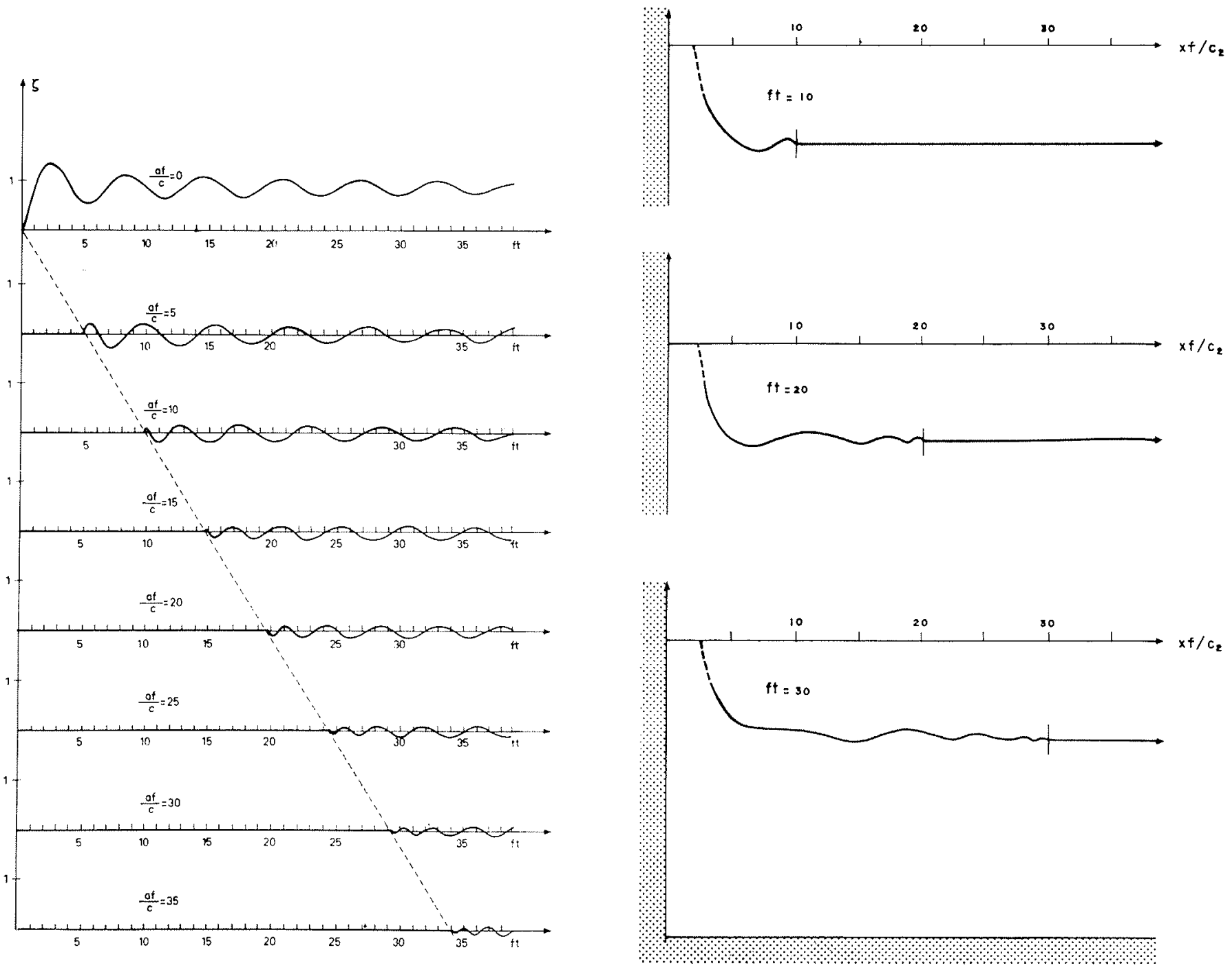

2/ Surélévation de la surface libre ou de l'interface en fonction du temps (ft) pour différentes distances à la côte (af/c) (cas d'un vent perpendiculaire à la côte lorsque les dissipations sont nulles).

3/ "Upwelling". Représentation de l'interface en fonction de la distance à la côte $\left(x f / c_{2}\right)$ à trois instants différents : $f t=10$, $\mathrm{ft}=20, \mathrm{ft}=30$ (vent parallèle à la côte, dissipations nulles). 
et $\vec{u}\left(x / c_{i}, t\right)=\frac{\tau_{j}}{\rho f} Y\left(t-x / c_{i}\right)\left[-\left(1-\cos f\left[t-x / c_{i}\right]\right) \vec{x}+\left(f\left[t-x / c_{i}\right]-\sin f\left[t-x / c_{i}\right]\right) \overrightarrow{\mathrm{y}}\right.$

$$
+\frac{x f}{c_{i}}\left(\left(1-\cos [f t] \vec{x}+(f t-\sin [f t]) \vec{y} \div \frac{J_{1} f \sqrt{t^{2}-x^{2} / c_{i}^{2}}}{f \sqrt{t^{2}-x^{2} / c_{i}^{2}}} Y\left(t-x / c_{i}\right)\right)\right]
$$

A partir des propriétés des transformées de Laplace (W. Sutton, 1934), on calcule facilement le développement asymptotique de $\vec{u}\left(x / c_{i}, t\right)$ pour $t$ grand $\left(t \gg x / c_{i}\right)$. On obtient :

$\vec{u}\left(x / c_{i}, t\right)=\frac{\tau_{u}}{\rho f}\left[\left(e^{-x f / c_{i}}+\cos [f t]\right) \vec{x}+\left(e^{-x f / c_{i}}[f t]-\sin [f t] \vec{y}+O\left(\frac{x f}{c_{i}} \frac{\sin (f t-\pi / 4) \vec{x}+\cos (f t-\pi / 4) \vec{y}}{(f t)^{1 / 2}}\right)\right]\right.$

$\vec{u}\left(x / c_{i}, t\right)$ croît linéairement comme $e^{-x j / c_{i}}(f t)$ lorsque $(f t)$ croît.

Les conditions océanographiques à la bouée-laboratoire du CNEXO, en été, donnent comme valeurs des différents paramètres :

$$
c_{1}=165 \mathrm{~m} / \mathrm{sec}, x f / c_{1}=0,07, \quad c_{2}=0,5 \mathrm{~m} / \mathrm{sec}, \quad x f / c_{2}=10, h_{1} /\left(h_{1}+h_{2}\right)=0,02
$$

Tant que le temps $t$ est inférieur à quelques périodes d'inertie, ce qui est un ordre de grandeur habituel pour les durées des perturbations météorologiques, le terme $\left[h_{1} /\left(h_{1}+h_{2}\right)\right] \vec{u}\left(x / c_{1}, t\right)$ est très petit devant $\vec{u}_{L}(t)$. Le terme $\vec{u}\left(x / c_{1}, t\right)$ perturbe très peu le courant dû au vent local.

Le terme $\left[h_{2} /\left(h_{1}+h_{2}\right)\right] \vec{u}\left(x / c_{2}, t\right)$ est nul pour $t<x / c_{2} ;$ un calcul complet de la forme (10) montre que ce terme est petit par rapport à $\vec{u}_{2}(t)$ tant que le temps $t$ est inférieur à quelques périodes d'inertie et que la forme asymptotique (11) est atteinte d'autant plus lentement que $x f / c_{2}$ est grand. On en conclut que le terme $\vec{u}\left(x / c_{2}, t\right)$ perturbe assez peu le courant dû au vent local pendant une durée qui va de l'instant initial jusqu'à plusieurs périodes d'inertie.

Lorsque le temps croît et devient supérieur à plusieurs périodes d'inertie, $\left[h_{2} /\left(h_{1}+h_{2}\right)\right] \vec{u}\left(x / c_{2}, t\right)$ devient important; on obtient alors comme forme asymptotique pour $\vec{u}_{1}(x, t)$ :

$u_{1}(x, t)=\frac{\tau_{y}}{\rho f}\left[\vec{x}+\left(\frac{h_{1}}{h_{1}+h_{2}} e^{-x f / c_{1}}+\frac{h_{2}}{h_{1}+h_{2}} e^{-x / c_{2}}\right)(-\vec{x}+[f t] \vec{y})+O\left(\frac{x f}{c_{2}} \frac{\sin (f t-\pi / 4) \vec{x}+\cos (f t-\pi / 4) \vec{y}}{(f t)^{1 / 2}}\right)\right]$

Il y a disparition des termes purement oscillatoires; le courant ne peut plus être relié au vent local.

La vitesse intégrée dans la couche inférieure est de la forme :

$$
\vec{u}_{2}(x, t)=\frac{h_{2}}{h_{1}+h_{2}}\left[\vec{u}\left(x / c_{1}, t\right)-\vec{u}\left(x / c_{2}, t\right)\right]
$$

Elle ne dépend plus du vent local mais uniquement des vitesses émises à la côte.

Remarque 1. - Les vitesses eulériennes les plus importantes sont situées dans le fluide supérieur; en effet, si l'on appelle $\vec{u}_{i}^{*}(x, t)$ la vitesse moyenne dans le milieu $i$, on a :

et

$$
\vec{u}_{1}^{*}(x, t)=\frac{1}{h_{1}} \vec{u}_{1}(x, t)
$$

$$
\vec{u}_{2} *(x, t)=\frac{1}{h_{2}} \vec{u}_{2}(x, t)
$$

ce qui donne :

$$
\left|\vec{u}_{1}^{*}(x, t)\right| /\left|\vec{u}_{2}^{*}(x, t)\right|=O\left(h_{2} / h_{1}\right)
$$

REMARQue 2. - Les raisonnements et les résultats sont analogues si l'on considère l'action d'un vent perpendiculaire au rivage. Seuls diffèrent les comportements asymptotiques; les vitesses sont toujours bornées lorsque $t$ tend vers l'infini. 


\section{Conclusion}

Lorsqu'il y a une thermocline bien formée, on peut considérer que l'océan se comporte comme un fluide à deux couches. Ce schéma rend compte d'une façon satisfaisante des ondes internes que l'on observe en été à la bouée-laboratoire du CNEXO, des upwellings côtiers et aussi de la présence d'oscillations d'inertie importantes sur les courants mesurés dans le fluide supérieur. On montre alors que le courant, en un point éloigné de la côte, est pratiquement égal au courant du vent local pour des durées qui vont de l'instant initial à quelques périodes d'inertie. Cette durée est une fonction croissante du rapport $x f / c_{2}$.

\section{Bibliographie}

CAHN (A.). - An investigation of the free oscillations of a simple current system (1945). J. Meteorology, 2, pp. 113-119.

CRÉPON (M). - Hydrodynamique marine en régime impulsionnel, $3^{\text {e }}$ partie: Océan à deux couches (1969). Cah. Océanogr., 21, pp. 863-877.

Crépon (M.), Gonella (J.), lacombe (H.), Stanislas (G.). - Participation française à l'opération Cofrasov I. Résultats préliminaires (1972). CNEXO (Résultats des campagnes à la mer), 4, pp. 91-106.

Crépon (M.) et ChOuChan (D.). - « Justification expérimentale, à partir des données des expériences Cofrasov et Coblamed, des théories des océans à deux couches 》 (1974) (à paraittre).

Gonella (J.), Crépon (M.) et Madelain (F.). - Observation de courant, vent et température à la bouée-laboratoire (position
A), septembre-octobre 1966. Cah. Océanogr., XXI, (9), nov. 1969, pp. 845-854. 1974.

Gonella (J.). - Effet d'écran d'une thermocline (1974). La Houille Blanche, $7 / 8$, .

Surron (W.). - The asymptotic expansion of a function whose operational equivalent is known (1934). J. London Math. Soc., 9, pp. 131-137.

\section{Discussion}

Président : M. le Professeur H. LACOMBE
M. le Président remercie M. CRÉpon de son intéressant exposé et ouvre la discussion.

M. Chabert-d'Hières suggère une explication à Ia direction des courants observés :

J'ai cru comprendre ce matin que, dans le cas de courants engendrés par le mistral, il y avait un phénomène d'《upwelling ». Par conséquent, on peut penser que les courants possèdent une certaine composante vers le large, à droite de la direction du vent, composante qui chasse les nappes d'eau chaude.

D'autres theories vont être testées, dans le Laboratoire de Grenoble, dans un canal de $8 \mathrm{~m}$ de long et $2 \mathrm{~m}$ de large, monté sur la plaque tournante, signale M. Chabert d'HiÈres.

Voilà un sujet passionnant pour une prochaine Session du Comité Technique de la S.H.F., conclut M. le Président, qui clôt la discussion et donne la parole à $\mathrm{M}$. ArHan pour l'exposé de la communication qu'il a établie en collaboration avec M. Cavanré (ce dernier, empêché, n’assiste pas à la séance) 